\title{
Molecular Effects of Glycerol on Lipid Monolayers at the Gas-Liquid Interface: Impact on Microbubble Physical and Mechanical Properties
}

\author{
Radwa H. Abou-Saleh, ${ }^{\dagger} \ddagger \odot$ James R. McLaughlan, ${ }^{\S}, \|$ Richard J. Bushby, ${ }^{\perp \odot}$ Benjamin R. Johnson, ${ }^{\dagger}$
} Steven Freear, ${ }^{\S}$ Stephen D. Evans, ${ }^{*, \dagger}$ and Neil H. Thomson ${ }^{*}, \dagger, \#(\mathbb{0}$

${ }^{\dagger}$ Molecular and Nanoscale Physics Group, School of Physics and Astronomy, University of Leeds, Leeds LS2 9JT, United Kingdom

${ }^{\ddagger}$ Biophysics Group, Department of Physics, Faculty of Science, Mansoura University, Mansoura, Egypt

${ }^{\S}$ School of Electronic and Electrical Engineering, University of Leeds, Leeds LS2 9JT, United Kingdom

"Leeds Institute of Medical Research, University of Leeds, St. James's University Hospital, Leeds LS9 7TF, United Kingdom

${ }^{\perp}$ School of Chemistry, University of Leeds, Leeds LS2 9JT, United Kingdom

${ }^{\#}$ Division of Oral Biology, School of Dentistry, University of Leeds, Leeds LS2 9LU, United Kingdom

Supporting Information

ABSTRACT: The production and stability of microbubbles (MBs) is enhanced by increasing the viscosity of both the formation and storage solution, respectively. Glycerol is a good candidate for biomedical applications of MBs, since it is biocompatible, although the exact molecular mechanisms of its action is not fully understood. Here, we investigate the influence glycerol has on lipidshelled MB properties, using a range of techniques. Population lifetime and single bubble stability were studied using optical microscopy. Bubble stiffness measured by AFM compression is compared with lipid monolayer behavior in a LangmuirBlodgett trough. We deduce that increasing glycerol concentrations enhances stability of MB populations through a 3-fold mechanism. First, binding of glycerol to lipid headgroups in the interfacial monolayer up to $10 \%$ glycerol increases $\mathrm{MB}$ stiffness but has limited impact on shell resistance to gas permeation and corresponding $\mathrm{MB}$ lifetime. Second, increased solution viscosity above $10 \%$ glycerol slows down the kinetics of gas transfer, markedly increasing MB stability.

Third, above $10 \%$, glycerol induces water structuring around the lipid monolayer, forming a glassy layer which also increases MB stiffness and resistance to gas loss. At $30 \%$ glycerol, the glassy layer is ablated, lowering the MB stiffness, but MB stability is further augmented. Although the molecular interactions of glycerol with the lipid monolayer modulate the MB lipid shell properties, MB lifetime continually increases from 0 to $30 \%$ glycerol, indicating that its viscosity is the dominant effect on $\mathrm{MB}$ solution stability. This three-fold action and biocompatibility makes glycerol ideal for therapeutic MB formation and storage and gives new insight into the action of glycerol on lipid monolayers at the gas-liquid interface.

\section{INTRODUCTION}

Microbubbles (MBs) for contrast enhanced ultrasound imaging are typically between 1 and $8 \mu \mathrm{m}$ in diameter and consist of a biocompatible lipid, protein, or polymer shell encapsulating a gas core, which is usually a perfluorocarbon. These MBs oscillate in response to ultrasound (US) excitation, and their high acoustic impedance mismatch with the surrounding tissue means that they act as efficient contrast agents for ultrasound imaging. ${ }^{1,2}$ Therapeutic MBs, in which the shell is functionalized to attach a drug payload and/or targeting agents, have been emerging as a potential route for targeted, triggered-release, drug delivery systems. ${ }^{3,4}$ Multifunctional $\mathrm{MB}$ coating has been used for magnetic resonance imaging contrast enhancement and gene delivery at the same time by coupling super-paramagnetic iron oxide nanoparticles with albumin shells. ${ }^{5,6}$ Typically, the MBs have a therapeutic agent conjugated to it, either in liposomes, ${ }^{7,8}$ oil nanodroplets, ${ }^{9}$ or genes attached to the MB shell, ${ }^{10,11}$ or in some cases the gas core itself may be therapeutic. ${ }^{12,13}$

For clinical and preclinical applications, control over MBs' size distribution, stability, and mechanical response to US are key parameters typically considered when optimizing $\mathrm{MB}$ production. These are controlled by the gas type, the MB shell, and the properties of the solution phase. The MB shell introduces a resistance to gas permeation and thus together

Special Issue: Microbubbles: Exploring Gas-Liquid Interfaces for Biomedical Applications

Received: December 12, 2018

Revised: $\quad$ March 22, 2019

Published: March 22, 2019 
with surface tension has an impact on $\mathrm{MB}$ size and lifetime. ${ }^{14-19}$ Phospholipid monolayers are a commonly used coating that has good shell resistance as well as a subharmonic response to low amplitude ultrasound. ${ }^{20,21}$ The gas core, and its solubility in the surrounding medium, ${ }^{22-24}$ also impact the MB lifetime with the use of higher-molecular-weight, lesssoluble perfluorocarbons (PFCs), leading to enhanced $\mathrm{MB}$ stability. ${ }^{17,22,25,26}$ Finally, the properties of the surrounding liquid medium are also important during storage or in vitro studies, where parameters such as temperature, pressure, viscosity, and concentration of the dissolved gas all play a role. $^{22,27}$

MBs have been widely investigated to increase their lifetime and stability in vitro and in vivo, either by, for example, altering the lipid shell composition ${ }^{28-30}$ or by the encapsulated gas. ${ }^{26,31}$ Recently in our group, we studied saturation of the surrounding medium with liquid PFC $\left(\mathrm{C}_{6} \mathrm{~F}_{10}\right)$ to increase the stability of MBs, and we showed that PFC molecules were incorporated in the shell lipid monolayer, which resulted in a $25 \%$ reduction in the surface tension that reduced the Laplace driving pressure for dissolution. ${ }^{22}$ Other groups have demonstrated that incorporation of viscosity agents within the production medium, such as $10 \%$ glycerol and $10 \%$ propylene glycol and $80 \%$ water, known as GPW, has an important role in producing smaller MBs and helps to control MB dispersity and stability. ${ }^{27,32-34}$ In a more detailed study, it was shown that varying solution viscosity using glycerol from 5 to $75 \mathrm{wt} \%$ using microfluidic electrohydrodynamic focusing production at voltages up to $12 \mathrm{kV}$ reduced $\mathrm{MB}$ size from 120 to $25 \mu \mathrm{m} .{ }^{35}$ In another study using a flow focusing PDMS chamber, glycerol and propylene glycol were used as the viscous agents to form $\mathrm{MBs}$ in solution and implicated as one of the factors to tailor MB size distribution. They reported that smaller MBs were produced only in the case of using $10 \mathrm{vol} \%$ viscous agents ${ }^{36}$ but not less. A similar effect was also noticed in the case of forming nanoemulsions with high concentrations of glycerol, up to $50 \%$, leading to $38 \%$ reduction in droplet size and narrower size distribution. ${ }^{37,38}$

Glycerol is a water miscible molecule that has been used for many years to stabilize the activity of enzymes and the native structure of proteins as well as enhancement of protein selfassembly. ${ }^{39,40}$ It has also been broadly used as a cryoprotectant to preserve the functionality of the biological molecules. ${ }^{41-43}$ Glycerol molecules contain only single bonds, making them flexible and able to adapt to optimize their hydrogen bonding with surrounding water. ${ }^{44}$ Phospholipids in water with higher concentrations of glycerol (10-30\%) have been shown to lead to the formation of so-called "glycerosomes," which have been shown to be more potent than aqueous liposomes for transdermal drug delivery. ${ }^{45,46}$ Glycerol is known to be a kosmotropic agent that increases water-water H-bonding in the solvation shell and diminishes the solubilization of hydrophobic groups, thus it has an ordering effect on lipids on proteins stabilizing the folded state ${ }^{39,47,48}$

There is ample evidence from the literature that sugars and sugar alcohols, such as glycerol, in solution affect the physical properties and the stability of lipid layers. ${ }^{49,50}$ The key reason glycerol and other sugars are used as cryo-preservatives is their ability to form glassy water-glycerol structures around biointerfaces during the process of freezing cells and liposomes, ${ }^{49-52}$ as well as forming water-glycerol bonding to stabilize proteins. ${ }^{39,53}$ These effects have also been used for prolonged stability of oil nanoemulsions $38,54,55$
A number of studies have investigated the influence of glycerol on different biological systems, and there have been debated conclusions regarding the exact mechanism and molecular behavior of glycerol at the membrane surface. Glycerol affects the viscosity of the solution, which is expected to influence the surface tension of the lipid membrane and alter its optimum curvature and solubility. ${ }^{48,56,57}$ One of the reported mechanisms refers to glycerol's ability to permeate the bilayer membrane and suppress intracellular ice formation during freeze and thaw cycles. ${ }^{41-43}$ An alternative hypothesis suggests that the similarity between water and glycerol leads to bonding with the lipid headgroup, which could suggest that glycerol partitions equally between the surface and the bulk. ${ }^{58}$ A thermodynamic study on DMPC vesicles demonstrated that glycerol has two competing effects at the molecular level, both partitioning to the membrane but also preferential exclusion from the interface. ${ }^{59}$ The second, possibly stronger, effect is that as glycerol concentration is increased the membrane hydration decreases. ${ }^{59}$

In spite of its popular use in lipid systems there are surprisingly fewer reports discussing the precise molecular effects of glycerol on lipid monolayers. In 2011, Pocivavsek et al. reported that glycerol not only has a fluidizing effect on model Lung surfactant monolayers but also leads to a stiffening at higher packing densities, ${ }^{62}$ which they attributed to the incorporation of glycerol molecules into the solvation shell, with the hypothesis that in the presence of glycerol the hydration shell retains two to three water molecules per lipid headgroup, increasing the stiffness of the monolayers. ${ }^{61,62}$

The aim of this study is to understand how glycerol influences the formation, stability, and mechanical properties of phospholipid coated MBs. This is the first detailed investigation of the effects of glycerol on lipid-shelled microbubbles, to attempt to deconvolute purely viscous effects from molecular interactions. Glycerol concentrations were varied between 1 and $30 \mathrm{vol} \%$ in the aqueous media during $\mathrm{MB}$ formation in a microfluidic device and subsequent storage in vials. Taken together, the results suggest complex and competing effects of glycerol at the molecular level, but the stability of the MBs always increases with concentration, even though the properties of the lipid layer, its hydration shell, and the properties of the bulk solution are in competition.

\section{EXPERIMENTAL SECTION}

Materials. Lipids used for MB formulations were 1,2-dipalmitoyl$s n$-glycero-3-phosphocholine (DPPC) and 1,2-distearoyl-sn-glycero-3phosphoethanolamine-N-[methoxy (polyethylene-glycol)-2000] (DSPE-PEG ${ }_{2000}$ ), purchased from Avanti Polar Lipids (Alabaster, AL, USA) and used without further purification. All lipids were received in powder form and dissolved in 50/50 chloroform/ methanol. Glycerol from Sigma-Aldrich (St. Louis, MO, USA) was used in the buffer surrounding the MBs. For testing MB lifetime, cell culture medium (RPMI from Invitrogen, Life Technologies, UK) was used with $10 \%(\mathrm{v} / \mathrm{v})$ fetal calf serum (Sigma-Aldrich, UK). The Neutravidin used for immobilizing MBs to surfaces for AFM measurements was obtained from Invitrogen, Life Technologies, UK.

Microbubble Production and Characterization. MBs were prepared as previously described. ${ }^{63,64}$ Briefly, the lipid shell was formulated with DPPC and $5 \mathrm{~mol} \%$ DSPE-PEG 2000 . The lipid mixture was dried under a steady stream of nitrogen gas on the vial walls. The dried film was then suspended in a solution containing 4 $\mathrm{mg} / \mathrm{mL} \mathrm{NaCl}$ and varying glycerol concentrations of $1,10,20$, and 30 vol \% (Sigma-Aldrich, UK) to a final lipid concentration of $1 \mathrm{mg} / \mathrm{mL}$. This solution was vortexed for $1 \mathrm{~min}$, then placed in an ultrasonic 
bath for $1 \mathrm{~h}$. The lipid solutions were allowed to cool down in the fridge at $4{ }^{\circ} \mathrm{C}$ for $5 \mathrm{~min}$ prior to use in the microfluidic-MB maker.

MBs were produced microfluidically according to our previously described protocols. ${ }^{64} \mathrm{C}_{3} \mathrm{~F}_{8}$ gas was used for the $\mathrm{MB}$ core, and the gas pressure was controlled using a Kukuke microprecision regulator (RS supplies, Leeds, UK). The liquid phase, containing the lipid mixture, was flow rate controlled using an Aladdin AL 2000 syringe pump (World Precision Instruments, Stevenage, UK).

For sizing and counting the produced MBs, a $10 \mu \mathrm{L}$ sample collected from the middle of the homogeneous $\mathrm{MB}$ solution was diluted 10 -fold. From this diluted sample, $30 \mu \mathrm{L}$ was introduced in a $50 \mathrm{~mm}$ depth chamber on a glass slide. MBs were allowed to rise for $\sim 2$ min before collecting images. An inverted microscope (Nikon, Japan) was used to image the MBs on a $60 \times$ magnification. A CCD camera (DS-Fil 5Mega pixel, Nikon, Japan) was used to capture 40 images for each sample, from which the concentration and size distribution were obtained using ImageJ freeware (http.//rsbweb.nih. gov/ij/) and analyzed using Origin Pro (Version 8.5 or later) to create the size distribution histograms.

Microbubble Stability. MB lifetime was measured as described previously. ${ }^{14}$ Briefly, $500 \mu \mathrm{L}$ of MB solution was introduced in $500 \mu \mathrm{L}$ of cell medium [RPMI from Invitrogen, Life Technologies, UK with $10 \%(\mathrm{v} / \mathrm{v})$ fetal calf serum (Sigma-Aldrich, UK)] and incubated at 37 ${ }^{\circ} \mathrm{C}$ in a digital dry block heater (Model D1100, Labnet International, USA). The vial containing the sample was left open exposed to air, and $10 \mu \mathrm{L}$ samples were collected every $15 \mathrm{~min}$ for sizing and counting. At each time point, the vial was closed and gently shaken, and a sample was collected. The MB samples were allowed 2 min to rise from the bulk to the top of the optical chamber, to ensure all bubbles in the sample were imaged and considered.

The shell resistance to gas permeation was monitored by following single MB dissolution as previously described. ${ }^{65}$ In brief, MBs were injected in a $50 \mathrm{~mm}$ deep fluid cell with fluid inlet and outlet ports and a replaceable glass slide top. These inlets were to allow degassed bubble solution to be flowed through the fluid cell containing the MBs under a flow rate of $2 \mathrm{~mL} / \mathrm{min}$. Several rinsing passages were used to ensure complete rinsing and buffer exchange takes place in the chamber. The change in bubble radius was then imaged with light microscopy.

AFM Mechanical Testing. For AFM testing, a diluted MB sample interacted with a biotinylated surface, to allow attachment of MB at the surface. For this, a 20 -nm-thick layer of gold with a $2.5 \mathrm{~nm} \mathrm{Cr}$ adhesion layer was deposited on a glass coverslip by thermal evaporation (Edwards Auto 306). The gold coverslips were incubated overnight in a 90:10 mol \% 6-mercaptohexanol (Sigma-Aldrich, Poole, UK)/biotinylated-thiol (Asemblon, WA, USA) ${ }^{60}$ solution to form a biotinylated self-assembled monolayer (SAM). The biotinylated SAM surface was incubated with $0.1 \mu \mathrm{M}$ Neutravidin for $\sim 1 \mathrm{~h}$ prior to adding the MBs. The sample was then placed into contact with the MB solution in an inverted configuration for $1 \mathrm{~h}$ to enable the buoyancy force to bind the MBs onto the surface. Repeat measurements with the AFM loading at the pole of the MB were performed as described previously ${ }^{14,63}$ using tip-less cantilevers (Nanosensors, UK). The cantilevers were calibrated using the thermal tuning method ${ }^{67}$ giving a spring constant $\sim 0.3 \mathrm{~N} / \mathrm{m}$. An MFP-3D AFM (Asylum Research, Santa Barbara, CA) was utilized to select a single $\mathrm{MB}$ within a small diameter range $(3-4 \mu \mathrm{m})$ for mechanical testing using the integrated optics of the AFM. The cantilever was then positioned above the pole of the $\mathrm{MB}$, and force curves were acquired in continuous mode. For all MB preparations, the loading velocity was fixed to $3 \mu \mathrm{m} / \mathrm{s}$, and force curves with a range of maximum applied loads were acquired: 5, 10, 30, and $50 \mathrm{nN}$. For each $\mathrm{MB}$ under investigation, 50 force-distance curves were collected with the cantilever in continuous mode to ensure reproducibility. Forcedistance curves were then converted to force-deformation curves with the AFM software accounting for the cantilever deflection and the calibrated deflection sensitivity.

Hysteresis between loading and unloading in the force-indentation curves collected has been observed for all samples and is related to the dissipation of energy during sample deformation. The viscoelastic behavior of the MBs is quantified by calculating the plasticity index $(\eta)$ from the hysteresis (ratio between the areas under the unloading $\left(A_{\mathrm{u}}\right)$ and loading curves $\left.\left(A_{\mathrm{L}}\right)\right), \eta=1-\left(A_{\mathrm{u}} / A_{\mathrm{L}}\right)$, which gives the relative plastic/elastic behavior of the material under force. For purely elastic samples, where $\eta=0$, the loading and unloading curves overlap, and the two areas are equal. For fully plastic properties, $\eta=1$, where the area under the unloading curve is zero, and the deformation of the MB remains constant when the load is removed. In our case, $\eta$ typically lies between 0 and 1 , representing a mixed viscoelastic behavior. ${ }^{63,68}$

Langmuir Isotherms. A Langmuir trough (KSV Nima) was used as previously described ${ }^{22}$ to measure the changes in monolayer compressability and elasticity. The trough was equipped with two movable PTFE barriers to compress the monolayer symmetrically. A Wilhelmy plate tensiometer (paper method) was used to measure the surface pressure of the monolayer. This experiment was performed for the same lipid monolayer composition used to produce MBs. A total of $20 \mu \mathrm{L}$ of a $1 \mathrm{mg} / \mathrm{mL}$ solution of DPPC $+5 \%$ DSPE-PEG $_{2000}$, in chloroform, was spread on the surface of the subphase. The subphase was mixed with MiliQ water at the same concentrations of glycerol as used for the MB productions.

\section{RESULTS AND DISCUSSION}

Viscosity agents are known to increase the lifetime of MBs and influence their size distributions. For the range of glycerol concentrations used here, the density and viscosity of the glycerol water mixtures have been calculated according to the formula and parameters published in 2008 by Cheng ${ }^{69}$ (Table $1)$. The results show that increasing the glycerol concentration does not affect the solution density significantly but has a large effect on the solution viscosity.

Table 1. Theoretical Calculations of the Density and Viscosity of the Prepared Solutions Surrounding the Bubbles and/or Used for the Langmuir Trough Subphase (Calculations Used the Parameters and Formulas in Cheng $2008^{69}$ )

$\begin{array}{ccc}\text { glycerol conc. \% } & \text { density }\left(\times 10^{3} \mathrm{~kg} / \mathrm{m}^{3}\right) & \text { viscosity }\left(\times 10^{-3} \mathrm{~Pa} \mathrm{~S}\right) \\ 0 & 1.00 & 1.00 \\ 1 & 1.00 & 1.03 \\ 10 & 1.03 & 1.34 \\ 20 & 1.05 & 1.75 \\ 30 & 1.07 & 2.24\end{array}$

Microbubble Production and Size Distribution. MBs were prepared with four different glycerol concentrations (1, $10,20$, and $30 \mathrm{vol} \%)$ in the surrounding medium. Figure 1a shows an example of a typical MB size distribution, for bubbles produced in the microfluidic "spray" regime. In this case, data are shown for the $1 \%$ solution, while histograms for the other solutions are presented in Figure $S 1$ and show a slight reduction in the modal size and the fwhm (Figure $1 \mathrm{~b}$ ). The average $\mathrm{MB}$ concentration produced in all cases was in the range of $(9 \pm 2) \times 10^{8} \mathrm{MB} / \mathrm{mL}$.

MB stability for the different glycerol concentrations was determined by measuring the $\mathrm{MB}$ concentration at different time points during incubation under physiological conditions, in cell medium, at $37^{\circ} \mathrm{C}$, in an open environment to allow gas exchange (Figure 2). There was no significant effect on MB population stability for glycerol concentrations less than $10 \%$, but considerable improvement in the lifetime was observed at $20 \%$ glycerol and higher. At 30\% glycerol, the whole population essentially remained stable over the time course of the experiment. This compares to a loss of $90 \%$ in the 

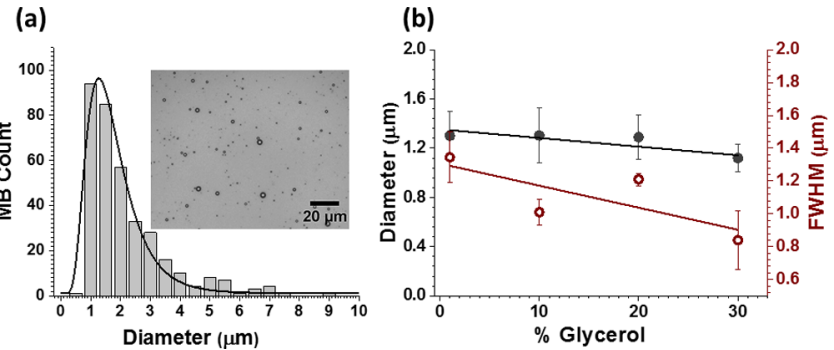

Figure 1. MB size distribution at increasing glycerol concentrations. (a) $\mathrm{MB}$ size distribution histogram in $1 \%$ glycerol. Inset: example optical image for the MB population. (b) MB modal diameter (filled circles) and the full width at half-maximum (FWHM; open circles) as a function of glycerol concentration, which shows a slight decrease in modal diameter with a more marked decrease in fwhm. Error bars represent standard deviation from four different repeats.

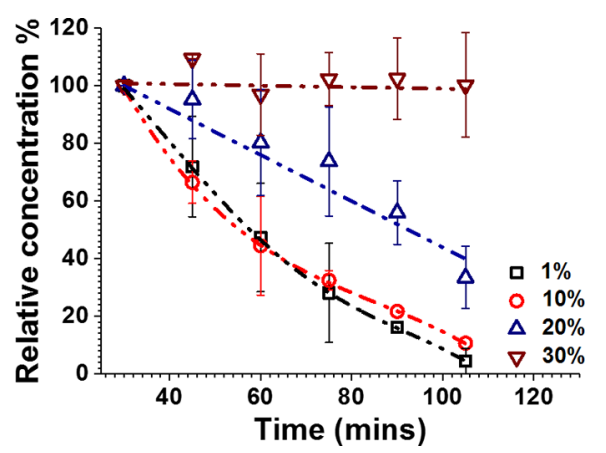

Figure 2. Lifetime of $\mathrm{C}_{3} \mathrm{~F}_{8} \mathrm{MB}$ with glycerol concentration in the surrounding $\mathrm{MB}$ solution. $\mathrm{MB}$ concentration decreased rapidly at the 1 and $10 \%$ glycerol concentration. The $20 \%$ concentration showed a slight improvement in MB lifetime. The 30\% glycerol sample showed considerable enhancement in the population lifetime during $100 \mathrm{~min}$ of observation. Error bars represent standard deviation from three different repeats.

population concentration with the lowest glycerol concentration of $1 \%$ within $\sim 100 \mathrm{~min}$. This effect is due to the glycerol directly influencing the organization in the lipid shell surrounding the $\mathrm{MB}$ and increasing resistance to permeation and thereby decreasing the rate of gas escape from the $\mathrm{MB}$ core to the surrounding medium. To address this, we also studied single bubble dissolution.

$\mathrm{MB}$ dissolution was investigated by following the $\mathrm{MB}$ size as a function of time, for individual MBs, across the glycerol concentration range (Figure 3a). For these studies, slightly larger MBs were considered to allow observation for prolonged periods. In all cases, the MBs initially decreased nearly linearly with time. Example images from the $10 \%$ sample are presented in Figure $3 \mathrm{~b}$ and show that MBs maintain their uniform circularity while the gas core escapes, indicating that lipids from the shell are shed during dissolution in a quasicontinuous manner. ${ }^{15}$ By contrast, the 20 and $30 \%$ samples show discrete steps in the single $\mathrm{MB}$ dissolution behavior and the MBs adopt noncircular and faceted shapes (Figure 3c). We have previously observed this effect for other stiff shelled MBs (e.g., actin coated MBs and for diacetylene polymerized $\mathrm{MB}$ shells) and have attributed this step like behavior to the stiffening of the MB coating. ${ }^{65}$

The effective $\mathrm{MB}$ shell resistance was determined by numerically fitting the experimental radius-time plots to the Epstein-Plesset equation by varying the shell resistance $R_{\text {shell }}$ (a)

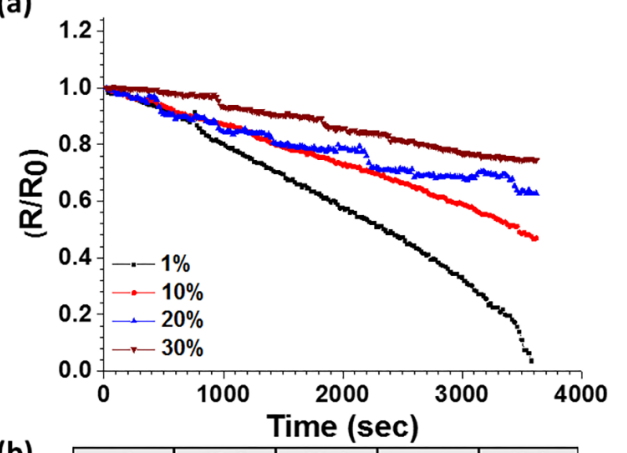

(b)

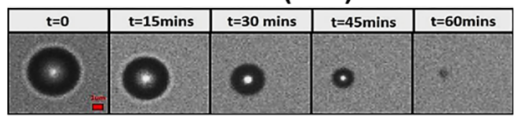

(c)

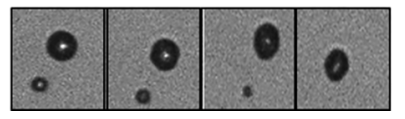

Figure 3. $M B$ gas dissolution. (a) Relative radius vs time plots presenting different $\mathrm{MB}$ dissolution as a function of time at different glycerol concentrations (average bubble size for all cases is $5.1 \pm 0.3$ ). (b) Selected images in the dissolution time course for MBs in $1 \%$ glycerol, showing that circularity is maintained during gas escape. (c) MBs for the $30 \%$ glycerol sample showing discrete shell cracking events and noncircularity of the bubbles corresponding to the observed steps in the time series. Scale bar $=1 \mu \mathrm{m}$.

for each case and assuming the following parameters: ${ }^{24}$ the saturation of the surrounding medium $(f)$ was $5 / 6$, and values of $5.2 \times 10^{-4}$ and $7.45 \times 10^{-4} \mathrm{~m}^{2} \mathrm{~s}^{-1}$ were used for the Ostwald coefficient $(H)$ and diffusivity of $\mathrm{C}_{3} \mathrm{~F}_{8}$ in water $\left(D_{\mathrm{w}}\right)$ as previously determined. ${ }^{15,24} \mathrm{We}$ also assumed that the surface tension $(\sigma)$ was constant throughout at a value appropriate for DPPC of $62 \mathrm{mN} / \mathrm{m}$ as determined by Borden et al. from measurements of the collapse pressure of a Langmuir monolayer. ${ }^{15}$

The average values calculated for the shell resistance, $R_{\mathrm{s}}$, were plotted for the different glycerol concentrations in Figure 4 and increased monotonically and in a superlinear manner with increasing glycerol concentration. The calculated $R_{s}$ values ranged from $(90 \pm 1) \times 10^{2} \mathrm{~s} / \mathrm{cm}$ for the $1 \%$ glycerol sample to $(340 \pm 2) \times 10^{2} \mathrm{~s} / \mathrm{cm}$ for the $30 \%$ glycerol sample. These values are 2 orders of magnitude higher than the values obtained by Heath et al., ${ }^{65}$ using an MB shell of DOPC/

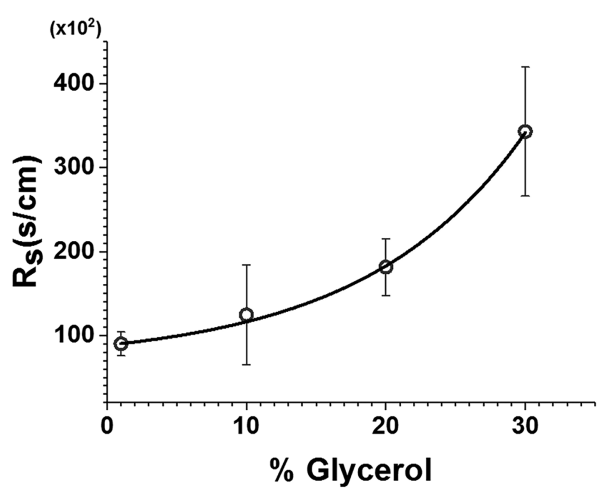

Figure 4. MB shell resistance as a function of glycerol concentration. Each value is the average of five to eight MBs, with an average size of $5.1 \pm 0.3 \mu \mathrm{m}$ diameter. The values are fitted to an exponential growth curve. Error bars represent standard deviation. 
DOTAP, and Borden et al., ${ }^{15}$ using a range of PC lipids and PEG40 stearate in the shell. This increase could be related to the different shell composition used here, which contains 5\% $\mathrm{PEG}_{2000}$-lipid, giving a thicker shell. In another study, Park et al. $^{70}$ tested different compositions of a diacetylene-PEG lipid MBs shell with and without polymerization in a $5 \%$ glycerol medium . The $R_{\mathrm{s}}$ values predicted for the unpolymerized MBs were $1 \times 10^{4} \mathrm{~s} / \mathrm{cm}$, which is the same order of magnitude to the values found here.

Microbubble Mechanical Properties. The mechanical properties of the $\mathrm{MBs}$ at each glycerol concentration were measured by AFM using a tip-less cantilever. MB stiffness was determined from the tangent of force-indentation data at different maximum applied loads, to understand the $\mathrm{MB}$ compression behavior with increasing pressure.

Figure 5a presents the characteristic compressibility behavior of $\mathrm{MBs}$ where $\mathrm{MB}$ stiffness increases with increasing the (a)
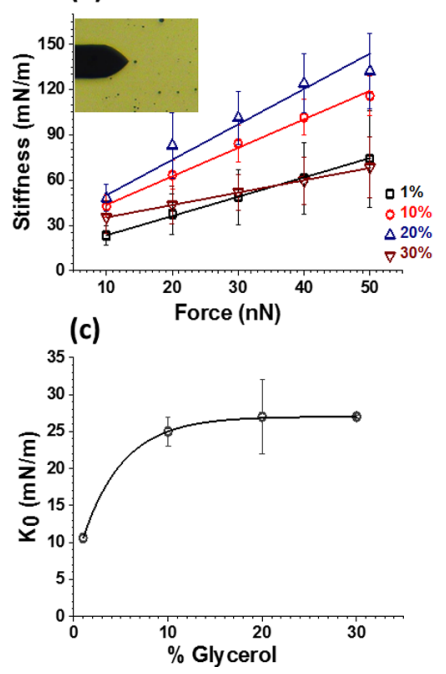

(b)

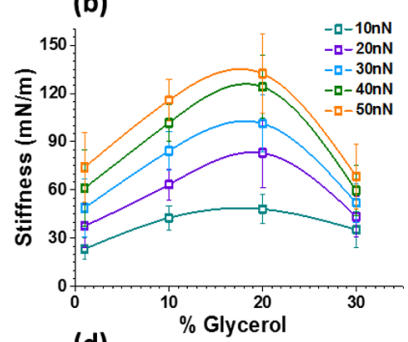

(d)

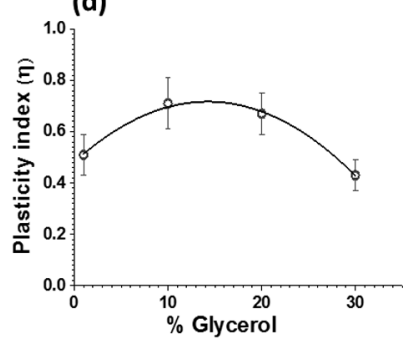

Figure 5. MB mechanical properties using AFM. (a) Compressibility behavior of MBs presented as stiffness measurements for MBs as a function of applied force at different glycerol concentrations. MB stiffness increases with increasing the applied force at each glycerol concentration. The overall $\mathrm{MB}$ stiffness increases with increased glycerol concentration from 1 to $20 \%$, with a different behavior at $30 \%$. The error bars show staandard deviation from averages of five to seven MBs in each case. The inset shows a typical example optical image of the AFM cantilever and MBs on the support surface. (b) MB stiffness as a function of glycerol concentration at all applied forces, showing the minimal change in stiffness at the minimum applied force, then peaks at $20 \%$ glycerol for all other forces. (c) Extrapolated stiffness data from a to show the effective spring constant of MBs at zero applied force. (d) Change in plasticity index values with glycerol concentration, showing a correlative behavior with $\mathrm{MB}$ stiffness in $\mathrm{b}$.

maximum applied load, for the different glycerol concentrations. All data sets show linearly increasing stiffness with applied load, and only for the $30 \%$ sample is the compressibility behavior (linear slope) different than all the other cases. Interestingly, for any given force, the stiffness was found to increase from $1 \%$ to $20 \%$ followed by a dramatic reduction in stiffness for the $30 \%$ samples (Figure $5 \mathrm{~b}$ ).

At low applied force $(10 \mathrm{nN}), A F M$ is assumed to probe predominantly the $\mathrm{MB}$ shell properties, which exhibit an increase up to $20 \%$ glycerol concentration. The pattern of behavior is the same across the range of applied forces but the variations of effective stiffness are larger at higher force. At these higher $\mathrm{MB}$ deformations, the effective stiffness of the whole bubble including the gas core is interrogated, rather than just the shell at low force $(10 \mathrm{pN})$.

Extrapolating the stiffness-force data (Figure 5a) to zero applied force yields the effective stiffness of $\mathrm{MBs}$, at zero deformation $\left(K_{0}\right)$. Figure $5 \mathrm{c}$ presents the average $K_{0}$ at each glycerol concentration. The value of $K_{0}$ increased rapidly from $11 \pm 0.6 \mathrm{mN} / \mathrm{m}$ at $1 \%$ glycerol to $25 \pm 2 \mathrm{mN} / \mathrm{m}$ at $10 \%$ glycerol and then essentially remained constant up to $30 \%$ glycerol. The observed jump in effective stiffness between 1 and $10 \%$ glycerol implies an interaction of the additional glycerol molecules with the lipid monolayer, stiffening the shell. These AFM shell stiffening data imply shell stiffening that also confirms the results from the dissolution experiment, Figure 4.

The plasticity index (PI) values for all samples was calculated and provides an indication of the viscoelastic behavior of the MBs (Figure 5d). At all glycerol concentrations, MBs exhibit viscoelastic behavior, with the highest plasticity occurring between 10 and $20 \%$ glycerol. These trends in MB stiffness and PI support the idea that a glassy waterglycerol hydration shell is forming between 10 and $20 \%$ glycerol, which is lost at the higher $30 \%$ concentration due to the formation of glycerosomes in the bulk solution (see discussion below).

Monolayer Isotherms. Langmuir isotherms of the DPPC + 5\% DSPE-PEG2000 lipid monolayers were collected on subphases of glycerol solutions, at concentrations ranging from 0 to $30 \mathrm{vol} \%$. Figure 6 presents the isotherms for increasing

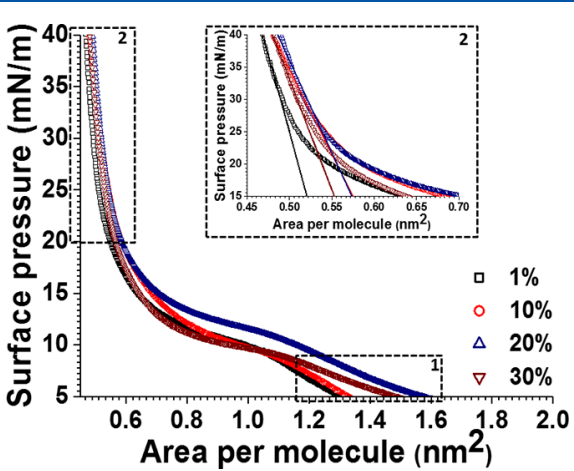

Figure 6. Pressure-area isotherms for monolayer of DPPC $+5 \%$ PEG2000 formed on subphases of glycerol concentrations 1-30\%. The inset is a zoom-in of region 2; this magnifies the liquid condensed phase (LC) to show the differences in the area per lipid molecule at each glycerol concentration. Region 1 is the lift-off area showing the initial interaction between molecules.

glycerol concentration at room temperature $\left(20^{\circ} \mathrm{C}\right)$. Region 1 indicates the initial area per lipid molecule, $A_{\mathrm{o}}$, at which the surface pressure rises above zero. The data (Figure S2) show that $A_{\mathrm{o}}$ increases with the glycerol concentration up to $20 \%$ followed by a decrease for the highest glycerol concentration of $30 \%$. This effect agrees with previous observations and interestingly mirrors the stiffness/plasticity trends seen in the AFM data. $44,59,71,72$

The inset in Figure 6 shows the liquid condensed phase (region 2), from which the area per lipid molecule is extrapolated from the isotherms. The area/molecule (at high surface pressure) and the slope of the tangent are used to estimate the isothermal compressibility, $C$, and the compres- 
sion modulus (elasticity), $K$, of the lipid modified interface using $^{22} C=-(1 / A(\mathrm{~d} \pi / \mathrm{d} A))$ and $K=1 / C$.

The results plotted in Figure 7 show that increasing glycerol concentration from 1 to $10 \%$ results in a slight increase in the

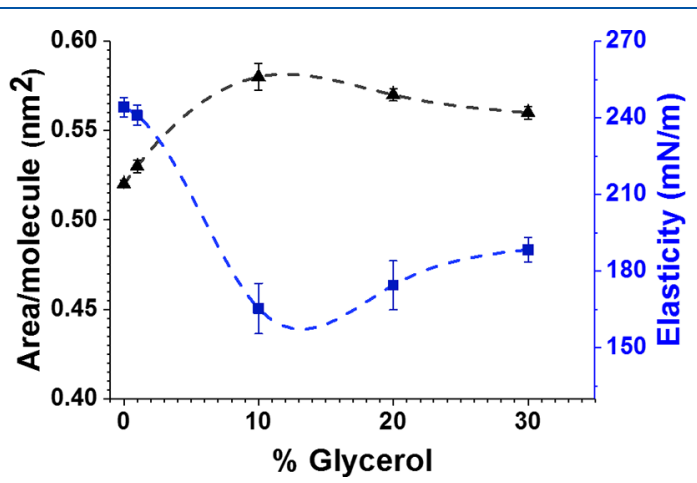

Figure 7. The influence of increasing glycerol concentration in the subphase on the area per lipid molecule (triangles) and the elasticity of the monolayer at the liquid-air interface in the LB trough (squares). These data were extracted from the lipid condensed phase area of the isotherms collected at different glycerol concentrations in the subphase. Error bars represent standard deviation from two repeats.

mean molecular area from $0.52 \pm 0.002$ to $0.58 \pm 0.007 \mathrm{~nm}^{2}$, which drops slightly as the glycerol concentration is increased to $30 \%$. For the $10 \%$ concentration, if the glycerol molecules insert into the $\mathrm{MB}$ shell, this has been estimated to be equivalent to one glycerol molecule for every five lipid molecules. These changes in area per molecule in the liquid condensed phase are accompanied by a decrease in monolayer compression elasticity $(k)$ from 242 to $147 \mathrm{mN} / \mathrm{m}$ with increasing glycerol concentration from 0 to $20 \%$. This is followed by an increase to $188 \mathrm{mN} / \mathrm{m}$ at $30 \%$ glycerol.

In addition to the other techniques, we have carried out initial ultrasound measurements of $\mathrm{MB}$ populations prepared with 1,20 , and $30 \%$ glycerol concentrations. The attenuation of the different $\mathrm{MB}$ populations (concentration of $2 \times 10^{6}$ $\mathrm{MB} / \mathrm{mL}$ ) was measured with a set of three matched pairs of unfocused transducers (V323, V384, V310, Olympus Industrial, Essex, UK), similar to the approach used before. ${ }^{8}$ These three transducers allowed for testing over a frequency range of $1.5-8.5 \mathrm{MHz}$ in $0.1 \mathrm{MHz}$ steps, with a peak acoustic pressure of $50 \mathrm{kPa}$. The acoustic attenuation as a function of frequency was used to estimate the shell parameters as shown in studies by Gorce et al. ${ }^{73}$ and Hoff. ${ }^{74}$ Preliminary results in Table S3 show that the shell stiffness peaks at $20 \%$ glycerol with the $30 \%$ values close to the $1 \%$ values. This trend is in agreement with both the AFM and LB trough data but will require more detailed investigation.

Glycerol has been used for a wide range of biological studies and bioinspired systems, mainly as a cryoprotectant, but the exact mechanism of glycerol on lipid membrane hydration and physical properties is still debated, mainly because it has more than one effect in any given system. It has been reported as having effects of both fluidizing and stiffening lipid membranes, while also dehydrating the lipid headgroup as well as forming structured glassy water-glycerol layers at interfaces. To further add to the complexity of behavior, it has been reported that above $10 \%$ glycerol, glycerosomes are formed with lipids.

On the basis of our results and current literature, we propose a working hypothesis for the molecular effects of glycerol on lipid-shelled MBs. For concentrations up to $10 \%$, the glycerol molecules bind to the lipid headgroup, increasing the area per molecule. At low surface pressures (region 1 in Figure 6), the glycerol is known to fluidize the membrane, ${ }^{62,72,75}$ but the lipid shell of the MBs will be in the liquid condensed phase (region 3 in Figure 6) where the binding of glycerol to the headgroups stiffens the membrane, which is what we observe in AFM compression measurements up to $20 \%$ glycerol. Glycerol binding to the lipids is more or less saturated at $10 \%$ glycerol (Figure 7). At the same time, glycerol-water glassy layers are forming around the lipid monolayer at the gas-liquid interface, increasing the effective stiffness of the MB coat. This effect continues up to $\sim 20 \%$ glycerol, beyond which glycerosomes start to form in solution. There is then a competition between use of glycerol to form these glassy layers and their disruption due to sequestering of glycerol into glycerosomes. By $30 \%$ glycerol, the glassy layer is all but abolished and glycerosomes dominate in solution. We now discuss in more detail how this picture aligns with the current literature and correlates with our measurements.

At low concentrations, up to $10 \%$, glycerol replaces water and binds to the phospholipid headgroup causing an increase in the area per molecule and increases the alkyl chain disorder. Furthermore, the ability of a glycerol molecule to hydrogen bond to more than one lipid molecule (its cross-linking ability) increases the stiffness of the layer. It also attracts and binds more water molecules forming the solvation (hydration) shell. Increasing the glycerol concentration in the range between 10 and $20 \%$ forms the more ordered glassy water-glycerol layer. Both these effects contribute toward increased shell resistance, which in turn reduces the gas exchange and increases $\mathrm{MB}$ stability (Figures 2 and 3). The observed increase in shell stiffness up to $20 \%$ glycerol, from Langmuir isotherms on monolayers (Figure 7) and from AFM mechanical testing on MBs (Figure $5 \mathrm{~b}$ ), is also consistent with the aforementioned cross-links and the glassy hydration layer. From molecular dynamics simulations of bilayers, ${ }^{76}$ it was suggested that at $15 \%$ mol concentration of glycerol, the lipid surface is enriched with glycerol molecules binding at a ratio of 2:1 glycerol per phospholipid headgroup, with hydrogen bonding between the phosphate moiety-glycerol or between two glycerol molecules, such that the lipid headgroup is dehydrated but yielding a reordering effect on the acyl chain ${ }^{76}$. This would make the shell stiffer and also increase resistance to permeation

At a high glycerol concentration $(>20 \%)$, two possible mechanisms could take place either alternatively or simultaneously. Considering that glycerol binding to the lipids is more or less saturated at $10 \%$ glycerol concentration. Glycerolwater glassy layers are formed at the lipid/aqueous interface, increasing the effective stiffness of the MB coat. This effect continues until $\sim 20 \%$ glycerol, beyond which glycerol as a cosolvent reduces the polarity of the mixture and alters the charge interaction between the lipid head groups, which facilitates the exposure of the hydrophobic tails to water ${ }^{48}$ and promotes the aggregation of the lipids to form micelles or glycerosomes ${ }^{38,57}$ that start to form in solution. This leads to a competition for the glycerol to form the glassy layers versus their sequestration into glycerosomes. By 30\% glycerol, the glycerosomes dominate in solution. Alternatively, glycerol molecules might sequester water molecules at the lipid interface and dehydrate the PEG chains, which will cause the PEG chains to aggregate and phase separate. This effect could explain the shell "cracking" behavior that was observed in the 
individual MBs dissolution experiments (Figure 3) and the unusual compressibility behavior in the AFM experiments for the $30 \%$ glycerol sample (Figure 5a), and in the associated Langmuir isotherms (Figure 6). It should also be noted that dehydration of lipid headgroups causes the area per lipid molecule to get smaller and increases ordering in the alkyl chains. The observed decrease in monolayer elasticity (Figure 7) could be attributed to the chain ordering effect of glycerol that favors the gel phase packing. ${ }^{47,59}$ In this phase, the repulsive interaction forces between the hydrophilic head groups increases, causing an increase in the free energy and the surface tension of the membrane. ${ }^{57,77,78}$ From the presented Langmuir isotherms, because of glycerol (surface tension 64 $\mathrm{mN} / \mathrm{m}^{79}$ ) incorporation in the lipid layer, the surface tension of the monolayer (using 10\% glycerol) is increased from 25 $\mathrm{mN} / \mathrm{m}^{24,80}$ to $31 \mathrm{mN} / \mathrm{m}$. At 20 and $30 \%$ glycerol, the fraction of glycerol incorporated in the shell is reduced again to be approximately 1 glycerol molecule for every 10 lipid molecules. Our estimates of glycerol to lipid molecular ratio are based on a simple calculation of glycerol molecular size and an assumption of intercalation in between the head groups. Molecular modeling and other studies indicate that one should expect a 1:1 ratio of glycerol to lipid at $10 \%$ glycerol and that glycerol binds to the lipid headgroups but does not insert between them.

The plasticity index derived from the AFM loading and unloading curves, follows a similar trend to that of the $\mathrm{MB}$ stiffness (Figure 5d). The increased plastic behavior at 10 and $20 \%$ appears related to the formation of the glassy glycerol hydration layer, as the $\mathrm{MBs}$ at $30 \%$ return to a similar viscoelasticity to that at $1 \%$. Again, this supports the idea of lipid dehydration at a high concentration of glycerol that can cause changes in phase transition and overall shell behavior.

It might be expected that changes in shell structure at high glycerol concentration should lead to a deterioration in $\mathrm{MB}$ lifetime; however, this was not observed and indicates there is another factor to be considered, which is the increased viscosity (from 1-2.24 Pa s) with increasing glycerol in solution from 1 to $30 \%$ (Table 1 ). Viscosity affects the mass transport kinetics at the membrane interface ${ }^{57}$ and leads generally to reduced gas exchange and increased bubble lifetime (Figures 2 to 4 ). It also alters the optimum monolayer curvature $^{57}$ and leads to a reduction in MB size and lower polydispersity of the microspray on chip production (Figure 1). A similar reduction of polydispersity index was reported by Saberi et al.; ${ }^{38,55}$ when producing oil nanoemulsions with $>20 \%$ glycerol concentrations, this effect was attributed to the tendency of glycerol to alter the interfacial tension, curvature, and characteristics of lipids ${ }^{48,56,57}$

Whether or not glycerosomes are forming in solution, we see that the increased concentration of glycerol leads to a tighter size distribution of $\mathrm{MBs}$; as a consequence, there is higher viscosity of the solution in the nozzle. There is a concomitant increase in the lifetime of the MBs, associated with a more stable bubble shell (lipid monolayer). Simple modeling with the Epstein-Plesset equation indicates that the effective shell resistance $\left(R_{\text {shell }}\right)$ goes up with increasing glycerol. In reality, it is likely that this is predominantly due to the diffusion coefficient of the gas going down in the higher viscosity solution since lifetime increases from 0 to $30 \%$. The molecular effects of glycerol could also contribute to increased shell resistance to gas permeation, at least up to $20 \%$ where glassy layers are formed.

\section{CONCLUSION}

Introducing glycerol into the solution during $\mathrm{MB}$ production and storage can greatly increase lifetime and reduce the polydispersity of the MB population. The multiple molecular effects of glycerol on the lipid monolayer at the gas-liquid interface can tune the physical and mechanical properties of lipid-shelled microbubbles. Despite modulation of the lipid monolayer properties through molecular interactions with glycerol, our results indicate that $30 \%$ glycerol is the optimal concentration for both $\mathrm{MB}$ formation and storage since it greatly enhances the lifetime and minimizes the polydispersity. One should bear in mind that once MBs are injected into the bloodstream, the in vivo solution environment will be very different; therefore higher concentrations of glycerol might be preferable for pure shelf life stability over optimization of shell properties. We expect that these findings may be relevant to MBs with different biomaterial shells, such as proteins, due to the molecular interactions of glycerol with water and biomolecules, as well as its dominant viscous behavior.

\section{ASSOCIATED CONTENT}

\section{S Supporting Information}

The Supporting Information is available free of charge on the ACS Publications website at DOI: 10.1021/acs.langmuir.8b04130.

Figure S1: Optical size distributions of MBs formed in 1-30\% glycerol. Figure S2: Area per lipid molecule calculated from Langmuir isotherms at high and low surface pressure for the lipid monolayers above an aqueous subphase of $1-30 \%$ glycerol. Table S1: MB shell stiffness and viscosity derived from ultrasound attenuation from $\mathrm{MB}$ populations for $1-30 \%$ glycerol (PDF)

\section{AUTHOR INFORMATION}

\section{Corresponding Authors}

*E-mail: S.D.Evans@leeds.ac.uk.

*E-mail: N.H.Thomson@leeds.ac.uk.

ORCID

Radwa H. Abou-Saleh: 0000-0002-8471-2659

Richard J. Bushby: 0000-0002-1627-6058

Steven Freear: 0000-0001-7858-4155

Neil H. Thomson: 0000-0001-7332-790X

\section{Notes}

The authors declare no competing financial interest.

\section{ACKNOWLEDGMENTS}

The authors would like to acknowledge EPSRC for funding (EP/I000623, EP/K023845) and thank the Microbubble Consortium (http://www.microbubbles.leeds.ac.uk/) at the University of Leeds for useful discussions. J.R.M. would like to acknowledge support from an EPSRC Innovation Fellowship (EP/S001069/1). The data presented in this article are openly available from the University of Leeds Data Repository (https://doi.org/10.5518/538).

\section{REFERENCES}

(1) Claudon, M.; Dietrich, C. F.; Choi, B. I.; Cosgrove, D. O.; et al. Guidelines and good clinical practice recommendations for contrast enhanced ultrasound (CEUS) in the liver - update 2012 a wfumbefsumb initiative in cooperation with representatives of afsumb, aium, 
asum, flaus and icus. Ultrasound in Medicine and Biology 2013, 39 (2), $187-210$.

(2) Cosgrove, D. Ultrasound contrast agents: An overview. Eur. J. Radiol. 2006, 60 (3), 324-330.

(3) Feinstein, S. B. The powerful microbubble: From bench to bedside, from intravascular indicator to therapeutic delivery system, and beyond. Am. J. Physiol Heart Circ Physiol 2004, 287 (2), H450-7.

(4) Lindner, J. R. Microbubbles in medical imaging: Current applications and future directions. Nat. Rev. Drug Discovery 2004, 3, 527-532.

(5) Guo, G. P.; Lu, L.; Yin, L. L.; Tu, J.; et al. Mechanical and dynamic characteristics of encapsulated microbubbles coupled by magnetic nanoparticles as multifunctional imaging and drug delivery agents. Phys. Med. Biol. 2014, 59 (22), 6729-6747.

(6) Guo, G. P.; Tu, J.; Guo, X. S.; Huang, P. T.; et al. Characterization of mechanical properties of hybrid contrast agents by combining atomic force microscopy with acoustic/optic assessments. J. Biomech 2016, 49 (3), 319-325.

(7) Geers, B.; Lentacker, I.; Sanders, N. N.; Demeester, J.; et al. Selfassembled liposome-loaded microbubbles: The missing link for safe and efficient ultrasound triggered drug-delivery. J. Controlled Release 2011, 152 (2), 249-56.

(8) Mclaughlan, J. R.; Harput, S.; Abou-Saleh, R. H.; Peyman, S. A.; et al. Characterisation of liposome-loaded microbubble populations for subharmonic imaging. Ultrasound in Medicine and Biology 2017, 43 (1), 346-356.

(9) Mico, V.; Charalambous, A.; Peyman, S. A.; Abou-Saleh, R. H.; et al. Evaluation of lipid-stabilised tripropionin nanodroplets as a delivery route for combretastatin A4. Int. J. Pharm. 2017, 526 (1-2), 547-555.

(10) Zhang, Q.; Wang, Z.; Ran, H.; Fu, X.; et al. Enhanced gene delivery into skeletal muscles with ultrasound and microbubble techniques. Acad. Radiol 2006, 13 (3), 363-7.

(11) Zhigang, W.; Zhiyu, L.; Haitao, R.; Hong, R.; et al. Ultrasoundmediated microbubble destruction enhances VEGF gene delivery to the infarcted myocardium in rats. Clin Imaging 2004, 28 (6), 395-8.

(12) Cavalieri, F.; Finelli, I.; Tortora, M.; Mozetic, P.; et al. Polymer microbubbles as diagnostic and therapeutic gas delivery device. Chem. Mater. 2008, 20, 3254-3258.

(13) Feshitan, J. A.; Legband, N. D.; Borden, M. A.; Terry, B. S. Systemic oxygen delivery by peritoneal perfusion of oxygen microbubbles. Biomaterials 2014, 35 (9), 2600-2606.

(14) Abou-Saleh, R. H.; Evans, S. D.; Thomson, N. H.; Swain, M. Polyethylene glycol lipid-shelled microbubbles: Abundance, stability, and mechanical properties. Langmuir 2014, 30, 5557-5563.

(15) Borden, M. A.; Longo, M. L. Dissolution behavior of lipid monolayer-coated, air-filled microbubbles: Effect of lipid hydrophobic chain length. Langmuir 2002, 18 (24), 9225-9233.

(16) De Jong, N.; Bouakaz, A.; Frinking, P. Basic acoustic properties of microbubbles. Echocardiography - A Journal of Cardiovascular Ultrasound and Allied Techniques 2002, 19 (3), 229-240.

(17) Kwan, J. J.; Borden, M. A. Lipid monolayer collapse and microbubble stability. Adv. Colloid Interface Sci. 2012, 183, 82-99.

(18) Overvelde, M.; Garbin, V.; Sijl, J.; Dollet, B.; et al. Nonlinear shell behavior of phospholipid-coated microbubbles. Ultrasound in Medicine and Biology 2010, 36 (12), 2080-2092.

(19) Sijl, J.; Dollet, B.; Overvelde, M.; Garbin, V.; et al. Subharmonic behavior of phospholipid-coated ultrasound contrast agent microbubbles. J. Acoust. Soc. Am. 2010, 128 (5), 3239-3252.

(20) De Jong, N.; Emmer, M.; Chin, C. T.; Bouakaz, A.; et al. "Compression-only" behavior of phospholipid-coated contrast bubbles. Ultrasound in Medicine and Biology 2007, 33 (4), 653-656.

(21) Frinking, P. A.; Gaud, E.; Brochot, J.; Arditi, M. Subharmonic scattering of phospholipid-shell microbubbles at low acoustic pressure amplitudes. IEEE Transactions on Ultrasonics Ferroelectrics and Frequency Control 2010, 57 (8), 1762-1771.

(22) Abou-Saleh, R. H.; Peyman, S. A.; Johnson, B. R. G.; Marston, G.; et al. The influence of intercalating perfluorohexane into lipid shells on nano and microbubble stability. Soft Matter 2016, 12 (34), $7223-7230$

(23) Katiyar, A.; Sarkar, K.; Jain, P. Effects of encapsulation elasticity on the stability of an encapsulated microbubble. J. Colloid Interface Sci. 2009, 336 (2), 519-25.

(24) Sarkar, K.; Katiyar, A.; Jain, P. Growth and dissolution of an encapsulated contrast microbubble: Effects of encapsulation permeability. Ultrasound Med. Biol. 2009, 35 (8), 1385-96.

(25) Rossi, S.; Waton, G.; Krafft, M. P. Phospholipid-coated gas bubble engineering: Key parameters for size and stability control, as determined by an acoustical method. Langmuir 2010, 26 (3), 16491655.

(26) Szijjarto, C.; Rossi, S.; Waton, G.; Krafft, M. P. Effects of perfluorocarbon gases on the size and stability characteristics of phospholipid-coated microbubbles: Osmotic effect versus interfacial film stabilization. Langmuir 2012, 28, 1182-1189.

(27) Talu, E.; Lozano, M. M.; Powell, R. L.; Dayton, P. A.; et al. Long-term stability by lipid coating monodisperse microbubbles formed by a flow-focusing device. Langmuir 2006, 22 (23), 94879490.

(28) Kooiman, K.; Kokhuis, T. J. A.; van Rooij, T.; Skachkov, I.; et al. DSPC or DPPC as main shell component influences ligand distribution and binding area of lipid-coated targeted microbubbles. Eur. J. Lipid Sci. Technol. 2014, 116 (9), 1217-1227.

(29) Kwan, J. J.; Borden, M. A. Lipid monolayer dilatational mechanics during microbubble gas exchange. Soft Matter 2012, 8 (17), 4756-4766.

(30) Mountford, P. A.; Sirsi, S. R.; Borden, M. A. Condensation phase diagrams for lipid-coated perfluorobutane microbubbles. Langmuir 2014, 30 (21), 6209-6218.

(31) Kabalnov, A.; Bradley, J.; Flaim, S.; Klein, D.; et al. Dissolution of multicomponent microbubbles in the bloodstream: 2. Experiment. Ultrasound Med. Biol. 1998, 24 (5), 751-760.

(32) Jiang, C. X.; Li, X.; Yan, F.; Wang, Z. H.; et al. Microfluidicassisted formation of multifunctional monodisperse microbubbles for diagnostics and therapeutics. Micro Nano Lett. 2011, 6 (6), 417-421.

(33) Kaya, M.; Gregory, T. S., V; Dayton, P. A. Changes in lipidencapsulated microbubble population during continuous infusion and methods to maintain consistency. Ultrasound Med. Biol. 2009, 35 (10), 1748-1755.

(34) Seo, M.; Gorelikov, I.; Williams, R.; Matsuura, N. Microfluidic assembly of monodisperse, nanoparticle-incorporated perfluorocarbon microbubbles for medical imaging and therapy. Langmuir 2010, 26 (17), 13855-13860.

(35) Parhizkar, M.; Stride, E.; Edirisinghe, M. Preparation of monodisperse microbubbles using an integrated embedded capillary T-junction with electrohydrodynamic focusing. Lab Chip 2014, 14 (14), 2437-2446.

(36) Talu, E.; Hettiarachchi, K.; Zhao, S.; Powell, R. L.; Lee, A. P.; Longo, M. L.; Dayton, P. A. Tailoring the size distribution of ultrasound contrast agents: Possible method for improving sensitivity in molecular imaging. Mol. Imaging 2007, 6 (6), 384-392.

(37) Qian, C.; McClements, D. J. Formation of nanoemulsions stabilized by model food-grade emulsifiers using high-pressure homogenization: Factors affecting particle size. Food Hydrocolloids 2011, 25, 1000-1008.

(38) Saberi, A. H.; Fang, Y.; McClements, D. J. Effect of glycerol on formation, stability, and properties of vitamin-E enriched nanoemulsions produced using spontaneous emulsification. J. Colloid Interface Sci. 2013, 411, 105-113.

(39) Gekko, K.; Timasheff, S. N. Mechanism of protein stabilization by glycerol - preferential hydration in glycerol-water mixtures. Biochemistry 1981, 20 (16), 4667-4676.

(40) Lee, J. C.; Timasheff, S. N. In vitro reconstitution of calf brain microtubules - effects of solution variables. Biochemistry 1977, 16 (8), $1754-1764$

(41) Alonso-Romanowski, S.; Biondi, A. C.; Disalvo, E. A. Effect of carbohydrates and glycerol on the stability and surface-properties of lyophilized liposomes. J. Membr. Biol. 1989, 108 (1), 1-11. 
(42) Mazur, P. Cryobiology - freezing of biological systems. Science 1970, 168 (3934), 939-949.

(43) Towey, J. J.; Dougan, L. Structural examination of the impact of glycerol on water structure. J. Phys. Chem. B 2012, 116 (5), 16331641.

(44) Egorov, A. V.; Lyubartsev, A. P.; Laaksonen, A. Molecular dynamics simulation study of glycerol-water liquid mixtures. J. Phys. Chem. B 2011, 115 (49), 14572-14581.

(45) Manca, M. L.; Cencetti, C.; Matricardi, P.; Castangia, I.; et al. Glycerosomes: Use of hydrogenated soy phosphatidylcholine mixture and its effect on vesicle features and diclofenac skin penetration. Int. J. Pharm. 2016, 511 (1), 198-204.

(46) Manca, M. L.; Zaru, M.; Manconi, M.; Lai, F.; et al. Glycerosomes: A new tool for effective dermal and transdermal drug delivery. Int. J. Pharm. 2013, 455 (1-2), 66-74.

(47) Koynova, R.; Brankov, J.; Tenchov, B. Modulation of lipid phase behavior by kosmotropic and chaotropic solutes - experiment and thermodynamic theory. Eur. Biophys. J. 1997, 25 (4), 261-274.

(48) Ruiz, C. C.; Diaz-Lopez, L.; Aguiar, J. Micellization of sodium dodecyl sulfate in glycerol aqueous mixtures. J. Dispersion Sci. Technol. 2008, 29, 266-273

(49) Crowe, J. H.; Crowe, L. M.; Carpenter, J. F.; Rudolph, A. S.; Wistrom, C. A.; Spargo, B. J.; Anchordoguy, T. J. Interactions of sugars with membranes. Biochim. Biophys. Acta, Rev. Biomembr. 1988, 947 (2), 367-384.

(50) Dashnau, J. L.; Nucci, N. V.; Sharp, K. A.; Vanderkooi, J. M. Hydrogen bonding and the cryoprotective properties of glycerol/ water mixtures. J. Phys. Chem. B 2006, 110 (27), 13670-13677.

(51) Dawidowski, J.; Bermejo, F. J.; Fayos, R.; Fernández Perea, R.; Bennington, S. M.; Criado, A. Coherent neutron scattering response from glassy glycerol. Phys. Rev. E: Stat. Phys., Plasmas, Fluids, Relat. Interdiscip. Top. 1996, 53 (5), 5079-5088.

(52) Doss, A.; Paluch, M.; Sillescu, H.; Hinze, G. From strong to fragile glass formers: Secondary relaxation in polyalcohols. Phys. Rev. Lett. 2002, 88 (9), No. 095701.

(53) Gekko, K.; Timasheff, S. N. Thermodynamic and kinetic examination of protein stabilization by glycerol. Biochemistry 1981, 20 (16), 4677-86.

(54) Anyarambhatla, G. R.; Needham, D. Enhancement of the phase transition permeability of dppc liposomes by incorporation of mppc: A new temperature-sensitive liposome for use with mild hyperthermia. J. Liposome Res. 1999, 9 (4), 491-506.

(55) Saberi, A. H.; Fang, Y.; McClements, D. J. Fabrication of vitamin e-enriched nanoemulsions: Factors affecting particle size using spontaneous emulsification. J. Colloid Interface Sci. 2013, 391, 95-102.

(56) D’Errico, G.; Ciccarelli, D.; Ortona, O. Effect of glycerol on micelle formation by ionic and nonionic surfactants at 25 degrees. $J$. Colloid Interface Sci. 2005, 286 (2), 747-754.

(57) Patel, H.; Raval, G.; Nazari, M.; Heerklotz, H. Effects of glycerol and urea on micellization, membrane partitioning and solubilization by a non-ionic surfactant. Biophys. Chem. 2010, 150, $119-128$.

(58) Schrader, A. M.; Cheng, C. Y.; Israelachvili, J. N.; Han, S. G. Communication: Contrasting effects of glycerol and DMSO on lipid membrane surface hydration dynamics and forces. J. Chem. Phys. 2016, 145 (4), No. 041101.

(59) Westh, P. Unilamellar dmpc vesicles in aqueous glycerol: Preferential interactions and thermochemistry. Biophys. J. 2003, 84, 341-349.

(60) Bianco, I. D.; Fidelio, G. D.; Maggio, B. Effect of glycerol on the molecular properties of cerebrosides, sulphatides and gangliosides in monolayers. Biochem. J. 1988, 251, 613-616.

(61) Krasteva, N.; Vollhardt, D.; Brezesinski, G.; Mohwald, H. Effect of sugars and dimethyl sulfoxide on the structure and phase behavior of DPPC monolayers. Langmuir 2001, 17 (4), 1209-1214.

(62) Pocivavsek, L.; Gavrilov, K.; Cao, K. D.; Chi, E. Y.; et al. Glycerol-induced membrane stiffening: The role of viscous fluid adlayers. Biophys. J. 2011, 101 (1), 118-127.
(63) Abou-Saleh, R. H.; Peyman, S. A.; Critchley, K.; Evans, S. D.; et al. Nanomechanics of lipid encapsulated microbubbles with functional coatings. Langmuir 2013, 29 (12), 4096-4103.

(64) Peyman, S. A.; Abou-Saleh, R. H.; McLaughlan, J. R.; Ingram, $\mathrm{N}$.; et al. Expanding 3D geometry for enhanced on-chip microbubble production and single step formation of liposome modified microbubbles. Lab Chip 2012, 12 (21), 4544-4552.

(65) Heath, G. R.; Abou-Saleh, R. H.; Peyman, S. A.; Johnson, B. R. G.; et al. Self-assembly of actin scaffolds on lipid microbubbles. Soft Matter 2014, 10, 694-700.

(66) Jeuken, L. J. C.; Daskalakis, N. N.; Han, X. J.; Sheikh, K.; et al. Phase separation in mixed self-assembled monolayers and its effect on biomimetic membranes. Sens. Actuators, B 2007, 124 (2), 501-509.

(67) Hutter, J. L.; Bechhoefer, J. Calibration of atomic-force microscope tips. Rev. Sci. Instrum. 1993, 64 (7), 1868-1873.

(68) Klymenko, O.; Wiltowska-Zuber, J.; Lekka, M.; Kwiatek, W. M. Energy dissipation in the AFM elasticity measurments. Acta Phys. Pol., A 2009, 115 (2), 548-551.

(69) Cheng, N. S. Formula for the viscosity of a glycerol-water mixture. Ind. Eng. Chem. Res. 2008, 47 (9), 3285-3288.

(70) Park, Y.; Luce, A. C.; Whitaker, R. D.; Amin, B.; et al. Tunable diacetylene polymerized shell microbubbles as ultrasound contrast agents. Langmuir 2012, 28, 3766-37772.

(71) Cadenhead, D. A.; Demchak, R. J. Observations and implications of glycerol-monomolecular film interactions. Biochim. Biophys. Acta, Lipids Lipid Metab. 1969, 176 (4), 849-57.

(72) Crowe, J. H.; Whittam, M. A.; Chapman, D.; Crowe, L. M. Interactions of phospholipid monolayers with carbohydrates. Biochim. Biophys. Acta, Biomembr. 1984, 769 (1), 151-159.

(73) Gorce, J. M.; Arditi, M.; Schneider, M. Influence of bubble size distribution on the echogenicity of ultrasound contrast agents - a study of sonovue (tm). Invest. Radiol. 2000, 35 (11), 661-671.

(74) Hoff, L. Acoustic Characterization of Contrast Agents for Medical Ultrasound Imaging; Norwegian University of Science and Technology: Trondheim, Norway, 2000.

(75) Cadenhead, D. A.; Bean, K. E. Selected lipid monolayers on aqueous -glycerol and aqueous urea substrates. Biochim. Biophys. Acta, Biomembr. 1972, 290, 43-50.

(76) Malajczuk, C. J.; Hughes, Z. E.; Mancera, R. L. Molecular dynamics simulations of the interactions of dmso, mono-and polyhydroxylated cryosolvents with a hydrated phospholipid bilayer. Biochim. Biophys. Acta, Biomembr. 2013, 1828, 2041-2055.

(77) Israelachvili, J. N.; Marcelja, S.; Horn, R. G. Physical principles of membrane organization. Q. Rev. Biophys. 1980, 13 (2), 121-200.

(78) Jahnig, F. What is the surface tension of lipid bilayer membrane? Biophys. J. 1996, 71, 1348-1349.

(79) Bandarkar, F.; Khattab, I. S.; Martinez, F.; Khoubnasabjafari, M.; et al. Viscosity and surface tension of glycerol+n-methyl-2pyrrolidone mixtures from 293 to 323K. Phys. Chem. Liq. 2015, 53 (1), 104-116.

(80) Katiyar, A.; Sarkar, K. Stability analysis of an encapsulated microbubble against gas diffusion. J. Colloid Interface Sci. 2010, 343 (1), 42-7. 